\title{
STABILITY ANALYSIS OF LINEAR MULTISTEP METHODS FOR DELAY DIFFERENTIAL EQUATIONS
}

\author{
V.L. BAKKE and Z. JACKIEWICZ \\ Department of Mathematical Sciences \\ University of Arkansas \\ Fayetteville, AR 72701, USA \\ (Received August 26, 1985 and in revised form January 28, 1986)
}

ABSTRACT. Stability properties of linear multistep methods for delay differential equations with respect to the test equation

$$
y^{\prime}(t)=a y(\lambda t)+b y(t), \quad t \geq 0,
$$

$0<\lambda<1$, are investigated. It is known that the solution of this equation is bounded if and only if $|a|<-b$ and we examine whether this property is inherited by multistep methods with Lagrange interpolation and by parametrized Adams methods.

KEY WORDS AND PHRASES: Linear multistep method, delay differential equation, stability analysis.

1980 AMS SUBJECT CLASSIFICATION CODE. 65L05.

\section{INTRODUCTION}

Consider the delay differential equation (DDE)

$$
\begin{aligned}
& y^{\prime}(t)=f(t, y(t), y(a(t))), \quad t \leq t_{0}, \\
& y(t)=g(t), \quad t_{0}-\tau \leq t \leq t_{0},
\end{aligned}
$$

$\tau \geq 0$, where $f:\left[t_{0}, \infty\right) \times R^{2} \rightarrow R$ and $a:\left[t_{0}, \infty\right) \rightarrow R$ are continuous and $t_{0}-\tau \leq a(t)$ $\leq t$. Such equations arise in a number of practical applications such as, for example, control theory, electrodynamics, viscoelasticity, biomathematics, and medical sciences (compare Hale [1]).

The convergence theory of numerical methods for DDEs is rather well developed and resembles the convergence theory of corresponding numerical methods for ordinary differential equations (ODEs) (see Tavernini [2-4], Jackiewicz [5-7], Zverkina [8]). In this paper we wish to concentrate on stability analysis of some methods for (1.1).

To investigate the stability properties of numerical methods for (1.1), these methods are usually applied, with a fixed positive step size $h$, to various test equations with known region of stability. The simplest test equation is

$$
\begin{array}{lr}
y^{\prime}(t)=q y(t-\tau), & t \leq 0, \\
y(t)=g(t), & -\tau \leq t \leq 0,
\end{array}
$$

$\tau \geq 0, q-$ real, and it is known (see Bellman and Cooke [9]) that the solution of 
this equation tends to zero as $t \rightarrow \infty$ for all $q$ if and only if $q \varepsilon(-\pi / 2 \tau, 0)$. The numerical method, with step size $h=\tau / \mathrm{m}$, where $\mathrm{m}$ is a positive integer, which inherits this property is said to be $\mathrm{DA}_{0}$-stable and Cryer [10] gives some necessary and sufficient conditions for linear multistep method to be $\mathrm{DA}_{0}-\mathrm{stable}$. He also introduced a more general notion of $\mathrm{GDA}_{0}$-stability which relaxes the condition $\mathrm{h}=\tau / \mathrm{m}$ to $h=\tau /(m-u)$, where $u \in[0,1)$, and investigated the properties of linear multistep methods with respect to this concept. Barwell [11] generalized some of Cryer's results to the case where $\mathrm{q}$ is complex and also considered a more general test equation

$$
\begin{array}{ll}
y^{\prime}(t)=p y(t)+q y(t-\tau), & t \geq 0, \\
y(t)=g(t), & -\tau \leq t \leq 0,
\end{array}
$$

with $\mathrm{p}$ and $\mathrm{q}$ complex. He introduced the notion of Q- and GQ-stability related to (1.2) (with $\mathrm{q}$ complex) and $\mathrm{P}$ - and GP- stability related to (1.3) which are analogues of Cryer's $\mathrm{DA}_{0}$ - and $\mathrm{GDA}_{0}-$ stability and investigated the stability properties of some simple multistep methods coupled with Lagrange interpolation. Still more general concepts of stability with respect to (1.2) were introduced by van der Houwen and Sommeijer [12] and stability criteria were derived for a class of linear multistep methods.

Stability analysis of numerical methods for DDEs is difficult since it is necessary to consider difference equations of arbitrarily high order. To illustrate this point suppose that the linear multistep method

$$
\begin{array}{lr}
\sum_{j=0}^{k} \alpha_{j} y_{h}\left(t_{i+k-j}\right)=h \sum_{j=0}^{k} \beta_{j} f\left(t_{i+k-j}, y_{h}\left(t_{i+k-j}\right), y_{h}\left(a\left(t_{i+k-j}\right)\right)\right), \\
y_{h}(s)=g_{h}(s), & s \varepsilon\left[t_{0}-\tau, t_{0}\right],
\end{array}
$$

$i=0,1, \ldots$, coupled with Lagrange interpolation of sufficiently high order, and step size $h=\tau / m$, where $m$ is a positive integer, is applied to (1.3). Here, $t_{i}=t_{0}$ $+i h, i=0,1, \ldots ; y_{h}$ is an approximate solution; and $g_{h}$ is an approximation to $g$. This leads to the difference equation

$$
\begin{array}{ll}
\sum_{j=0}^{k} \alpha_{j} y_{h}\left(t_{i+k-j}\right)=h \sum_{j=0}^{k} \beta_{j}\left(p y_{h}\left(t_{i+k-j}\right)+q y_{h}\left(t_{i+k-j-m}\right)\right), & s \varepsilon\left[t_{0}-\tau, t_{0}\right],
\end{array}
$$

$i=0,1, \ldots$, of order $m+k$. Now in order to establish some stability properties of (1.4) we should be able to decide whether or not the solution $y_{h}$ of (1.5) is bounded (or tends to zero as $t \rightarrow \infty$ ). This is, in general, a nontrivial task and satisfactory results were obtained only for simplest numerical methods. Barwel1 [11] established Pand GP-stability for first and second order backward differentiation methods and Jackiewicz [13] determined stability regions for $\theta$-methods. Similar results with respect to the test equation (1.2) were obtained by Cryer [10] and Barwe11 [11]. Cryer proved that the $\Theta$-methods are $\mathrm{DA}_{0}-\mathrm{stable}$ if and only if $\Theta \varepsilon\left[\frac{1}{2}, 1\right]$ and that the Backward Euler method and the trapezoidal method are $\mathrm{GDA}_{0}-\mathrm{stable}$. Barwell proved that the Backward Euler method is Q-stable and conjectured that it is GQ-stable. For more general methods some stability results were established by Wiederholt [14], Al-Mutib [15], and Oppelstrup [16]. Wiederholt determined numerically (via boundary locus 
method) the set of all (hp,hq) such that $y_{h}$ (given by (1.5)) tends to zero as $t \rightarrow \infty$ for second order Milne predictor-corrector method and third order Adams predictor-corrector method for $m=1,2$, and 3 . Similar results were obtained by $A 1-$ Mutib for the Runge-Kutta-Merson method, the trapezium rule and the fourth order implicit Runge-Kutta method. Oppelstrup investigated stability properties of Runge-Kutta-Fehlberg method combined with Hermite-Birkhoff interpolation with respect to the test equation (1.2).

More general test equations for functional differential equations were considered by Bickart [17] and Brayton and Willoughby [18].

It is the purpose of this paper to present stability analysis of some linear multistep methods based on the test equation

$$
\begin{aligned}
& y^{\prime}(t)=a y(\lambda t)+b y(t), \quad t \geq 0, \\
& y(0)=y_{0},
\end{aligned}
$$

where $a, b$, and $\lambda$ are real and $0<\lambda<1$. The importance of such equations in practical applications is discussed, for example, by Fox et al [19]. It follows from the results of Kato and McLeod [20] (see also Fox et a1 [19]) that all solutions of (1.6) are bounded if and only if $|a|<-b$ and we investigate whether this property is inherited by the approximate solution $y_{h}$ when (1.4) is applied to (1.6). The problem is that the application of (1.4) to (1.6) leads to difference equations which are not of fixed order. A a consequence, the approach of Barwel1 [11], Cryer [10], Jackiewicz [13] is not applicable (compare the discussion of this topic in Jackiewicz [13]), and a different method of attack is needed. Roughly speaking, the approach of this paper consists of the following. Assuming that $y_{h}\left(t_{j}\right)$ for $j \leq i$ are bounded by a constant $M$ and $\left\|y_{h}\right\|_{\left[t_{0}, t_{i}\right]}$ is bounded by $\gamma M$ for some $\gamma \geq 1$, we are looking for conditions such that $y_{h}\left(t_{i+1}\right)$ and $\left\|y_{h}\right\|_{\left[t_{i}, t_{i+1}\right]}$ are bounded by the same constants $M$ and $\gamma M$ respectively. Denoting the set of all (hb,ha) for which this is true by $A_{\gamma}$, we obtain a lower bound on the stability region with respect to (1.6) in the form $\bigcup_{\gamma>1} A_{\gamma}$. This approach works reasonably well for simple multistep methods such as, for example $\theta$-methods. We were also able to obtain some sufficient stability conditions for linear multistep methods with Lagrange interpolation (Section 2) and for low order parametrized Adams-Bashforth and Adams-Moulton methods (Section 3). Unfortunately, this approach does not carry over to general linear multistep methods with Hermite interpolation and high order parametrized Adams methods.

As a byproduct of our analysis we established that the stability region for $\theta-$ methods with respect to (1.3) is contained in the stability region with respect to (1.6). This partially answers the conjecture posed in Jackiewicz [13].

2. STABILITY ANALYSIS OF LAGRANGE INTERPOLATORY EXTENSIONS OF LINEAR MULTISTEP METHODS

A Lagrange interpolatory extension of the linear multistep method for ODEs with coefficients $\alpha_{j}, \beta_{j}, j=0,1, \ldots, k$, is the method defined by

and

$$
\sum_{j=0}^{k} \alpha_{j} y_{h}\left(t_{i+k-j}\right)=h \sum_{j=0}^{k} \beta_{j} F\left(t_{i+k-j}\right),
$$

$$
y_{h}\left(t_{i+k-1}+r h\right)=\sum_{j=0}^{k} U_{j}(r) y_{h}\left(t_{i+k-j}\right),
$$


$i=0,1, \ldots, r \in[0,1]$, where $F\left(t_{v}\right)=f\left(t_{v}, y_{h}\left(t_{v}\right), y_{h}\left(a\left(t_{v}\right)\right)\right)$ and (2.2) is Lagrange interpolation formula, i.e. $U_{0}(1)=1, U_{j}(1)=0, j=1,2, \ldots, k, U_{1}(0)=1, U_{j}(0)=$ $0, j \neq 1$; and $\sum_{j=0} U_{j}(r)=1$ (compare Tavernini [3], where a more general Hermite interpolatory extension is defined). This method can be written in the form

$$
a_{0} y_{h}\left(t_{i+k-1}+r h\right)+\sum_{j=1}^{k} a_{j}(r) y_{h}\left(t_{i+k-j}\right)=h \sum_{j=0}^{k} b_{j}(r) F\left(t_{i+k-j}\right) \text {, }
$$

$i=0,1, \ldots, \quad r \varepsilon[0,1]$, where $a_{0}=\alpha_{\lambda} ; \quad \alpha_{j}(r)=\alpha_{j} U_{0}(r)-\alpha_{0} U_{j}(r), j=1,2, \ldots, k ; \quad b_{j}(r)=$ $\beta_{j} U_{0}(r), j=0,1, \ldots, k$. Denote by $y_{h}^{\lambda}$ the approximate solution obtained when the method (2.3) is applied to (1.6). We propose the following definition.

DEFINITION. For given values of $x=h b$ and $y=h a$, the method (2.3) is said to be absolutely stable, if $y_{h}^{\lambda}$ is bounded for $0<\lambda<1$. A region of absolute stability is the set of all points ( $x, y)$ such that the method (2.3) is absolutely stable. The method (2.3) is said to be A-stable if the region of absolute stability includes the stability region for $(1.6)$, i.e. the set $\{(x, y):|y|<-x\}$.

We have the following:

THEOREM 1. Assume that $(x, y)$ satisfies the inequality

$$
\sum_{j=0}^{k}\left|-\alpha_{j}+x \beta_{j}\right|+\gamma|y| \sum_{j=0}^{k}\left|\beta_{j}\right| \leq\left|\alpha_{0}-x \beta_{0}\right|,
$$

where $r=\sup \left\{\sum_{j=0}^{k}\left|U_{j}(r)\right|: \quad r \in[0,1]\right\}$. Then the method (2.3) is absolutely stable.

PROOF. It ${ }^{j}=0$ clear from (2.2) that if $\left|y_{h}^{\lambda}\left(t_{j}\right)\right|$ is bounded by a constant $M$ for $j \leq i$ then $\left\|y_{h}^{\lambda}\right\|_{\left[0, t_{i}\right]}$ is bounded by $\gamma M$. Here, $\left\|y_{h}\right\|_{\left[0, t_{i}\right]}=\sup \left\{\left|y_{h}(s)\right|: s \varepsilon\left[0, t_{i}\right]\right\}$. Assume that $\left|y_{h}\left(t_{j}^{\lambda}\right)\right| \leq M$ for $j \leq i$. Then it is easy to check, using (2.1) with

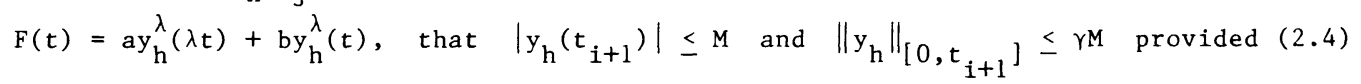
holds, and the proof is complete.

To illustrate this theorem we apply it now to some simple methods for DDEs.

EXAMPLE 1. Consider the $\theta$-methods with linear interpolation for DDEs (1.1). These are the methods of the form

$$
\begin{aligned}
& y_{h}\left(t_{i+1}\right)=y_{h}\left(t_{i}\right)+r h\left[\theta F\left(t_{i+1}\right)+(1-\theta) F\left(t_{i}\right)\right], \\
& y_{h}\left(t_{i}+r h\right)=(1-r) y_{h}\left(t_{i}\right)+r y_{h}\left(t_{i+1}\right),
\end{aligned}
$$

$i=0,1, \ldots, r \in[0,1]$. Now $r=1$ and the inequality (2.4) takes the form

$$
|1+(1-\theta) x|+|y| \leq|1-\theta x| \text {. }
$$

It is easy to verify that the solution of this inequality is $R_{1}$ for $0 \leq \theta \leq \frac{1}{2}$ and $R_{1} \cup R_{2}$ for $\frac{1}{2}<\theta \leq 1$, where

and

$$
R_{1}=\{(x, y):|y| \leq-x \text { and }|y| \leq 2+(1-2 \theta) x\}
$$

$$
R_{2}=\{(x, y):|y| \leq-2+(2 \theta-1) x\} .
$$

(In particular, the Backward Euler method $(\theta=1)$ is A-stable). Comparing this with Jackiewicz [13], p.391, we see that the stability region of $\theta$-methods with respect to (1.3) is contained in the stability region of these methods with respect to (1.6), which partially answers the conjecture posed in Jackiewicz [13]. 
EXAMPLE 2. Consider the trapezoidal method with quadratic interpolation $y_{h}\left(t_{i}+r h\right)=\frac{1}{2}(r+1)(2-r) y_{h}\left(t_{i}\right)+\frac{r}{2}(r-1) y_{h}\left(t_{i+1}\right)+\frac{r}{4}(r+1) h\left[F\left(t_{i+1}\right)+F\left(t_{i}\right)\right]$, $i=0,1, \ldots, \quad r \in[0,1]$. This method can be written in the form

$$
\begin{gathered}
y_{h}\left(t_{i+1}\right)=y_{h}\left(t_{i}\right)+\frac{h}{2}\left[F\left(t_{i+1}\right)+F\left(t_{i}\right)\right], \\
y_{h}\left(t_{i}+r h\right)=\frac{r}{2}(r+1) y_{h}\left(t_{i+1}\right)+\left(1-r^{2}\right) y_{h}\left(t_{i}\right)+\frac{r}{2}(r-1) y_{h}\left(t_{i-1}\right), \\
i=0,1, \ldots, \quad r \varepsilon[0,1] \text { (see Tavernini [3]). Now } \gamma=\frac{5}{4} \text { and the inequality (2.4) reads } \\
\left|1+\frac{1}{2} x\right|+\frac{5}{4}|y| \leq\left|1-\frac{1}{2} x\right| .
\end{gathered}
$$

The solution of this inequality is the set

$$
R=\left\{(\mathrm{x}, \mathrm{y}): \quad|\mathrm{y}| \leq-\mathrm{x} \text { and }|\mathrm{y}| \leq \frac{8}{5}\right\} .
$$

For comparison, for the trapezoidal rule with linear interpolation (this corresponds to $\theta=\frac{1}{2}$ in Example 1) the solution of (2.4) is

$$
R^{*}=\{(\mathrm{x}, \mathrm{y}):|\mathrm{y}| \leq-\mathrm{x} \text { and }|\mathrm{y}| \leq 2\} \text {, }
$$

and $R \subset R^{*}$. This suggest that the trapezoidal rule with linear interpolation may have better stability properties than trapezoidal rule with quadratic interpolation.

EXAMPLE 3. Consider the method

$$
y_{h}\left(t_{i-1}+r h\right)=y_{h}\left(t_{i-2}\right)+(1+r) h F\left(t_{i}\right)
$$

$i=0,1, \ldots, \quad r \in[0,1]$ or, equivalently

$$
\begin{gathered}
y_{h}\left(t_{i}\right)=y_{h}\left(t_{i-2}\right)+2 h F\left(t_{i}\right), \\
y_{h}\left(t_{i-1}+r h\right)=\frac{1+r}{2} y_{h}\left(t_{i}\right)+\frac{1-r}{2} y_{h}\left(t_{i-2}\right),
\end{gathered}
$$

$i=0,1, \ldots, r \in[0,1]$. This method is not of type (2.3), but the same approach can be used to obtain a sufficient condition for absolute stability. It is clear that $\gamma=1$ and inequality (2.4) takes the form

$$
1+2|y| \leq|1-2 x|
$$

Therefore, this method is absolutely stable if $(x, y) \varepsilon A_{1} \cup A_{2}$, where

$$
\begin{aligned}
& A_{1}=\{(x, y):|y| \leq-x\}, \\
& A_{2}=\{(x, y):|y| \leq x-1\} .
\end{aligned}
$$

In particular, it is A-stable.

EXAMPLE 4. Consider the backward differentiation methods:

$$
\begin{gathered}
\sum_{j=0}^{k} \alpha_{k, j} y_{h}\left(t_{i+k-j}\right)=h F\left(t_{i+k}\right), \\
y_{h}\left(t_{i+k-1}+r h\right)=\sum_{j=0}^{k} U_{k, j}(r) y_{h}\left(t_{i+k-j}\right),
\end{gathered}
$$

$i=0,1, \ldots, \quad r \in[0,1], k=1,2, \ldots, 6$, where

$$
\begin{aligned}
& \alpha_{k, 0}=\sum_{i=1}^{k} \frac{1}{i}, \quad \alpha_{k, j}=\frac{(-1)^{j}}{j}\left(\begin{array}{l}
k \\
j
\end{array}\right), \quad j=1,2, \ldots, k, \\
& U_{k, j}(r)=\prod_{\substack{m=0 \\
m \neq j}}^{k} \frac{r+m-1}{m-j}, \quad k=1,2, \ldots, \quad j=1,2, \ldots, k
\end{aligned}
$$


(the case $k=1$ corresponds to $\theta=1$ in Example 1). Now inequality (2.4) reads

$$
\gamma_{k}:=\sup \left\{\sum_{j=0}^{k}\left|\alpha_{k, j}\right|: \quad r \quad \begin{array}{l}
\sum_{j=1}^{k}\left|\alpha_{k, j}\right|+\gamma_{k}|y| \leq\left|\alpha_{k, 0}-x\right|, \\
[0,1]\}, \text { and its solution is } \\
A_{k}=A_{k, 1} \cup A_{k, 2},
\end{array}\right.
$$

where

$$
\begin{aligned}
& A_{k, 1}=\left\{(x, y): \quad x+\gamma_{k}|y| \leq \sum_{j=1}^{k} \frac{1}{j}\left(1-\left(\begin{array}{l}
k \\
j
\end{array}\right)\right)\right\}, \\
& A_{k, 2}=\left\{(x, y): \quad x-\gamma_{k}|y| \leq \sum_{j=1}^{k} \frac{1}{j}\left(1+\left(\begin{array}{l}
k \\
j
\end{array}\right)\right)\right\}
\end{aligned}
$$

(note that $\sum_{j=1}^{k} \frac{1}{j}\left(1-\left(\begin{array}{c}k \\ j\end{array}\right)\right)<0$ for $\left.k \geq 2\right)$. Unfortunately, this approach cannot be used to determine the stability of these methods in the neighborhood of the origin for $k \geq 2$.

3. STABILITY ANALYSIS OF PARAMETRIZED ADAMS METHODS

We will consider the (explicit) Adams-Bashforth and the (implicit) Adams-Moulton formulas. The Adams-Bashforth methods are given by

$$
y_{h}\left(t_{i+k-1}+r h\right)=y_{h}\left(t_{i+k-1}\right)+h \sum_{j=0}^{k} b_{k, j}(r) F\left(t_{i+k-1-j}\right),
$$

$i=0,1, \ldots, \quad r \in(0,1]$, where

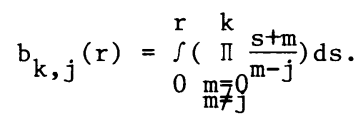

The method (3.1) can also be written in the form

$$
y_{h}\left(t_{i+k-1}+r h\right)=y_{h}\left(t_{i+k-1}\right)+h \sum_{j=0}^{k} c_{j}(r) \nabla^{j} F\left(t_{i+k-1}\right) \text {, }
$$

$i=0,1, \ldots, \quad r \varepsilon(0,1]$, where $\nabla^{j}$ is the backward difference operator of order $j$. The coefficients $c_{j}, j=0,1, \ldots, k$, are independent of $k$, and are given by

$$
c_{j}(r)=(-1) \int_{0}^{j}\left(\begin{array}{c}
-s \\
m
\end{array}\right) d s \text {. }
$$

Using the generating function

we also have

$$
G(t, r):=\sum_{m=0}^{\infty} c_{m}(r) t^{m}=\frac{1-(1-t)^{-r}}{\ln (1-t)}
$$

$$
c_{0}(r)=r, \quad \sum_{j=0}^{m} \frac{1}{j+1} c_{m-j}(r)=(-1)^{m+1}\left(\begin{array}{c}
-r \\
m+1
\end{array}\right) .
$$

Expressing $\nabla^{j} F\left(t_{i+k-1}\right)$ in (3.3) in terms of $F\left(t_{i+k-1-v}\right)$ and comparing (3.3) and (3.1) we can easily find the following relationship between $b_{k, j}$ and $c_{j}$ :

$$
b_{k, j}(r)=(-1)^{j} \sum_{m=j}^{k}\left(\begin{array}{l}
m \\
j
\end{array}\right) c_{m}(r) \text {. }
$$

Consider now the Adams-Moulton formulas for DDEs. These methods read

$$
y_{h}\left(t_{i+k-1}+r h\right)=y_{h}\left(t_{i+k-1}\right)+h \sum_{j=0}^{k} b_{k, j}^{*}(r) F\left(t_{i+k-j}\right) \text {, }
$$

$i=0,1, \ldots, \quad r \in(0,1]$, where

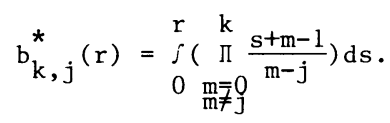


Another representation of $(3.6)$ is

$$
\left.y_{h}\left(t_{i+k-1}+r h\right)=y_{h}\left(t_{i+k-1}\right)+h \sum_{j=0}^{k} c_{j}^{*}(r) j_{F(t} t_{i+k}\right),
$$

$i=0,1, \ldots, \quad r \varepsilon(0,1]$, where the coefficients $c_{j}^{\star}, j=0,1, \ldots, k$. are given by

$$
c_{j}^{*}(r)=(-1)^{j} \int_{-1}^{-1+r}\left(\begin{array}{c}
-s \\
j
\end{array}\right) d s .
$$

Using the generating function

$$
G^{*}(t, r)=\sum_{m=0}^{\infty} c^{*}(r) t^{m}=\frac{(1-t)-(1-t)^{1-r}}{\ln (1-t)}
$$

we also have

$$
c_{0}^{*}(r)=r, \quad \sum_{j=0}^{m} \frac{1}{j+1} c_{m-j}^{*}(r)=(-1)^{m-1}\left(\begin{array}{c}
1-r \\
m+1
\end{array}\right)
$$

(compare Tavernini [3]). The relationship between $b_{k, j}^{*}$ and $c_{j}^{*}$ is

$$
b_{k, j}^{*}(r)=(-1)^{j} \sum_{m=j}^{k}\left(\begin{array}{l}
m \\
j
\end{array}\right) c_{m}^{*}(r) .
$$

Now we list some properties of the coefficients of the Adams-Bashforth and Adams-Moulton methods which will be useful later on. To be consistent with Henrici [21] we will use the following notation:

$$
\beta_{k, j}:=b_{k, j}(1), \quad \gamma_{j}:=c_{j}(1), \quad \beta_{k, j}^{*}:=b_{k, j}^{*}(1), \quad \gamma_{j}^{*}:=c_{j}^{*}(1) \text {. }
$$

P1. $\quad \gamma_{\mathrm{m}}>0, \quad \mathrm{~m} \leq 0$.

P2. $\quad \gamma_{0}^{*}=1, \quad \gamma_{\mathrm{m}}^{*}<0, \quad \mathrm{~m} \geq 1$.

P3. $\sum_{i=0}^{\mathrm{m}} \gamma_{i}^{*}=\gamma_{\mathrm{m}}, \quad \mathrm{m} \geq 0$.

P4. $\quad \beta_{k, 0}>0, \quad k \geq 0$.

P5. $\quad u_{k}:=\beta_{k, 0}-\sum_{j=1}^{k}\left|\beta_{k, j}\right|>0, \quad k=0,1,2 ; \quad u_{k}<0, \quad k \geq 3$.

P6. $\quad \beta_{k, 0}^{*}>0, \quad \beta_{k, 1}^{*}>0, \quad k \geq 1$.

P7. $\beta_{k, 1}^{*}-\beta_{k, 0}^{*}>0, \quad k \geq 1$.

P8. $\quad w_{k}^{*}:=\beta_{k, 0}^{*}-\sum_{j=1}^{k}\left|\beta_{k, j}\right|=0, \quad k=1 ; \quad w_{k}^{*}<0, \quad k \geq 2$.

P9. $u_{k}^{*}:=\beta_{k, 0}^{*}+\beta_{k, 1}^{*}-\sum_{j=2}^{k}\left|\beta_{k, j}^{*}\right|>0, \quad k=1,2,3,4,5 ; \quad u_{k}^{*}<0, \quad k \geq 6$.

P10. $\left\|b_{k, j}\right\|_{[0,1]}=\left|b_{k, j}(1)\right| ; \quad\left\|b_{k, j}^{*}\right\|_{[0,1]}=\left|b_{k, j}(1)\right|, \quad k \geq 0, \quad j=0,1, \ldots, k$.

The properties Pl-P3 are well known (compare Henrici [21]), where the first few values of $\gamma_{m}$ and $\gamma_{m}^{*}$ are also listed, or Hall [22]). P4 follows from (3.5) for $r=1, j=0$, and P1. It is easy to check, using (3.5) for $r=1$, that

$$
u_{k}=\sum_{m=0}^{k} \gamma_{m}-\sum_{m=0}^{k} \underset{m=j}{\sum} \underset{j}{m} \gamma_{m}=1+\sum_{m=1}^{k} \gamma_{m}-\sum_{m=1}^{k} \underset{j=1}{\sum} \underset{j}{m} \gamma_{m}=1+\underset{m=1}{k}\left(2-2^{m}\right) \gamma_{m} .
$$

Hence, $u_{0}=1, u_{1}=1, u_{2}=\frac{1}{6}, u_{3}=-\frac{25}{12}$, and $u_{k+1}<u_{k}$ for $k \geq 3$ which establishes P5. P6 follows from (3.10) for $r=1, j=0,1$, and P1-P3. Using (3.10) for $\mathrm{r}=1$ we obtain

$$
\beta_{k, 1}^{*}-\beta_{k, 0}^{*}=-\sum_{i=2}^{k}(1+i) \gamma_{i}^{*}
$$


which, together with P2, establishes P7. In riew of (3.10) for $r=1$ we have

$$
\begin{aligned}
& \mathrm{w}_{\mathrm{k}}^{*}=\sum_{\mathrm{m}=0}^{\mathrm{k}} \gamma_{\mathrm{m}}^{*}+\sum_{\mathrm{m}=1}^{\mathrm{k}} \mathrm{m} \gamma_{\mathrm{m}}^{*}+\sum_{j=2}^{\mathrm{k}} \underset{\mathrm{m}=\mathrm{j}}{\mathrm{k}}\left({ }_{\mathrm{j}}^{\mathrm{m}}\right) \gamma_{\mathrm{m}}^{*}=\gamma_{0}^{*}+2 \gamma_{1}^{*}+\sum_{\mathrm{m}=2}^{\mathrm{k}}(1+\mathrm{m}) \gamma_{\mathrm{m}}^{*} \\
& +\sum_{m=2}^{k} \sum_{j=2}^{k}\left({ }_{j}^{m}\right) \gamma_{m}^{*}=\sum_{m=2}^{k}\left(1+m+2^{m}-m-1\right) \gamma_{m}^{*}=\sum_{m=2}^{k} 2^{m} \gamma_{m}^{*}
\end{aligned}
$$

which proves P8. Similarly,

$$
\mathrm{u}_{\mathrm{k}}^{*}=1+\sum_{\mathrm{m}=2}^{\mathrm{k}}\left(2^{\mathrm{m}}-2 \mathrm{~m}\right) \gamma_{\mathrm{m}}^{*} \text {. }
$$

It is clear that $u_{k}^{*}>0$ for $k=1,2,3,4,5, u_{6}^{*}<0$ and $u_{k+1}^{*}<u_{k}^{*}$ for $k \geq 6$ which proves P9. P10 follows easily from (3.2) and (3.7).

We are now in a position to formulate our stability theorems.

THEOREM 2. Denote by $A_{\gamma}$ the set of all $(x, y)=(h b, h a)$ such that

$$
\left|1+\beta_{k, 0} x\right|+\sum_{j=1}^{k}\left|\beta_{k, j}\right||x|+\gamma \sum_{j=0}^{k}\left|\beta_{k, j}\right||y| \leq 1
$$

and

$$
\sum_{j=0}^{k}\left|\beta_{k, j}\right|(|x|+\gamma|y|) \leq \gamma-1,
$$

and put $A=\bigcup_{Y \geq 1} A_{\gamma}$. Then if $(x, y) \varepsilon A$ the Adams-Bashforth method (3.1) is absolutely stable.

PROOF. The method (3.1) applied to (1.6) takes the form

$$
y_{h}^{\lambda}\left(t_{i+k-1}+r h\right)=y_{h}^{\lambda}\left(t_{i+k-1}\right)+h \sum_{j=0}^{k} b_{k, j}(r)\left(a y_{h}^{\lambda}\left(\lambda t_{i+k-1-j}\right)+b y_{h}^{\lambda}\left(t_{i+k-1-j}\right)\right),
$$

$i=0,1, \ldots, \quad r \varepsilon(0,1]$. Let $M$ be a constant such that $\left\|y_{h}^{\lambda}\right\|_{\left[0, t_{k-1}\right]} \leq M$. Assume

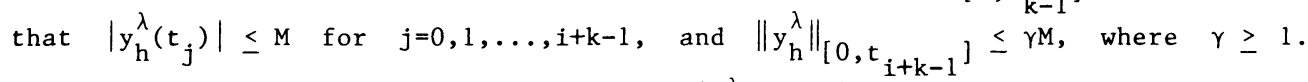
Then, using (3.13) for $r=1$ it follows that $\left|y_{h}^{\lambda}\left(t_{i+k}\right)\right| \leq M$ if (3.11) is satisfied. Similarly, using (3.13) and P10 we can see that $\left\|y_{h}^{\lambda}\right\|_{\left[0, t_{i+k}\right]} \leq \gamma M$ if (3.12) is satisfied. Therefore, if $(\mathrm{x}, \mathrm{y}) \varepsilon \mathrm{A}$, the approximate solution $\mathrm{y}_{\mathrm{h}}^{\lambda}$ given by (3.13) is bounded and the theorem follows. Define $u_{k}$ as in P5 and put $v_{k}:=\sum_{j=0}^{k}\left|\beta_{k, j}\right|$. It is easy to see, using P4, that $A_{\gamma}$ can be written in the form

where

$$
A_{\gamma}=B_{\gamma} \cap C_{\gamma}
$$

and

$$
B_{\gamma}=\left\{(x, y): \quad x \leq 0, \quad u_{k} x+\gamma v_{k}|y| \leq 0, \quad v_{k}(-x+\gamma|y|) \leq 2\right\}
$$

$$
C_{\gamma}=\left\{(x, y): \quad x \leq 0, \quad v_{k}(-x+\gamma|y|) \leq \gamma-1\right\} .
$$

It is clear in view of P5 that $B_{\gamma}$ is empty for $k \geq 3$, therefore this theorem applies only for $k=0,1$, and 2. It follows after some computations that the boundary of $A$ for $k=0,1$, and 2 is given by

$$
y=\left\{\begin{array}{lr} 
\pm\left(2+v_{k} x\right) /\left(3 v_{k}\right), & -2 / v_{k} \leq x \leq-1 / \beta_{k}, 0 \\
\pm u_{k} x /\left(v_{k}\left(2 \beta_{k, 0} x-1\right)\right), & -1 / \beta_{k, 0} \leq x \leq 0 .
\end{array}\right.
$$


These boundaries for $y \geq 0$ are plotted in Fig. 1 .

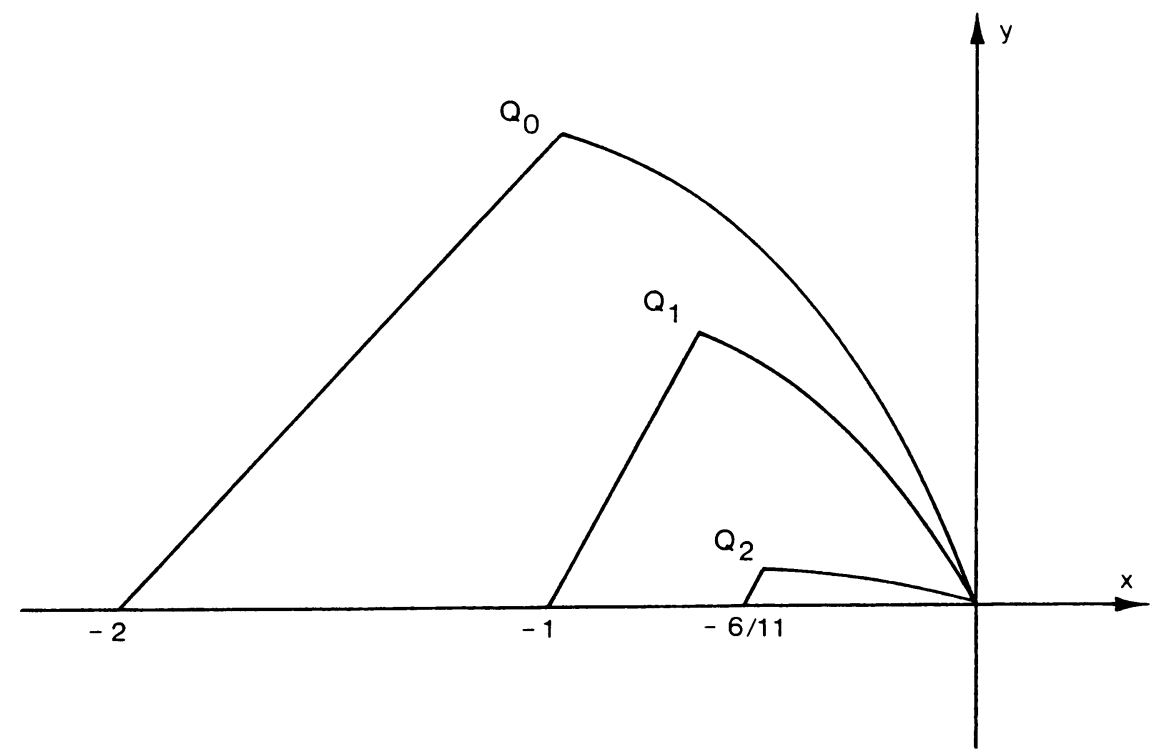

Fig. 1. Stability regions for Adams-Bashforth methods for $k=0,1,2$.

The coordinates of the points $Q_{i}\left(x_{i}, y_{i}\right), i=0,1,2$, are given in Table 1 below.

\begin{tabular}{ccc}
\hline $\mathrm{k}$ & $\mathrm{x}_{\mathrm{k}}$ & $\mathrm{y}_{\mathrm{k}}$ \\
\hline 0 & -1 & $1 / 3$ \\
1 & $-2 / 3$ & $1 / 4$ \\
2 & $-12 / 23$ & $2 / 253$ \\
\hline & Table 1 &
\end{tabular}

For $k=0$ we already obtained a better bound on stability region in Example 1 for $\theta=0$. Our next theorem deals with the Adams-Moulton methods (3.6).

THEOREM 3. Denote by $A_{\gamma}^{*}$ the set of all $(x, y)=(h b, h a)$ such that

$$
\left|1+\beta_{k, 1}^{*} x\right|+\sum_{j=2}^{k}\left|\beta_{k, j}^{*}\right||x|+\gamma \sum_{j=0}^{k}\left|\beta_{k, j}^{*}\right||y| \leq\left|1-\beta_{k, 0}^{*} x\right|
$$

and

$$
\sum_{j=0}^{k}\left|\beta_{k, j}^{*}\right|(|x|+\gamma|y|) \leq \gamma-1,
$$

and put $A^{*}=\underset{\gamma \geq 1}{\bigcup_{1} A_{\gamma}}$. Then if $(x, y) \in A^{*}$ the Adams-Moulton method (3.6) is absolutely stable.

PROOF. The proof is similar to that of Theorem 2. The method (3.6) applied to (1.6) takes the form

$$
y_{h}^{\lambda}\left(t_{i+k-1}+r h\right)=y_{h}^{\lambda}\left(t_{i+k-1}\right)+h \sum_{j=0}^{k} b_{k, j}^{*}(r)\left(a y_{h}^{\lambda}\left(\lambda t_{i+k-j}\right)+b y_{h}^{\lambda}\left(t_{i+k-j}\right)\right),
$$

$i=0,1, \ldots, \quad r \in(0,1]$. For $r=1$ this can be written in the form

$$
\begin{aligned}
\left(1-h b \beta_{k, 0}^{*}\right) y_{h}^{\lambda}\left(t_{i+k}\right) & =\left(1+h b \beta_{k, 1}^{*}\right) y_{h}^{\lambda}\left(t t_{i+k-1}\right)+h a \sum_{j=0}^{k} \beta_{k, j}^{*} y_{h}^{\lambda}\left(\lambda t{ }_{i+k-j}\right) \\
& +h b \sum_{j=2}^{k} \beta_{k, j}^{*} y_{h}^{\lambda}\left(t_{i+k-j}\right) .
\end{aligned}
$$


Denote by $i_{0}$ the smallest integer such that $t_{i_{0}+k} \leq t_{i_{0}+k}$ and let $M$ be a

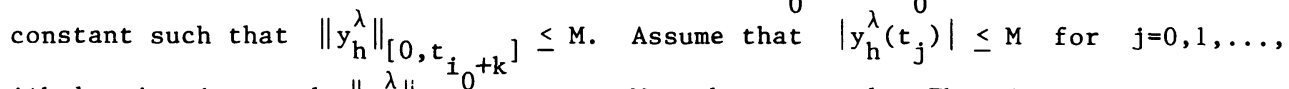
$i+k-1, \quad i \geq i_{0}$, and $\left\|y_{h}^{\lambda}\right\|_{\left[0, t_{i+k-1}\right]} \leq \gamma M$, where $\gamma \geq 1$. Then it is easy to check using (3.17) that $\left|y_{h}\left(t_{i+k}\right)\right| \leq M$ if (3.14) is satisfied. Similarly, using (3.16) and P10 it follows that $\left\|y_{h}\right\|_{\left[0, t_{i+k}\right]} \leq \gamma M$ if (3.15) holds. Therefore, if $(x, y) \varepsilon A^{*}$, the approximate solution $y_{h}^{\lambda}$ defined by (3.16) is bounded, which is our claim.

For $k=0$ (this corresponds to the backward Euler method) condition (3.14) and (3.15) take the form

$$
\begin{aligned}
& 1+\gamma|y| \leq|1-x|, \\
& |x|+\gamma|y| \leq \gamma-1,
\end{aligned}
$$

and it can be verified that the boundary of the region $A^{*}$ is given by

$$
y= \begin{cases} \pm x /(2 x-1), & x \leq 0 \\ \pm(x-2) /(2 x-1), & x \geq 2\end{cases}
$$

We obtained a better bound on stability region in Example 1 for $\theta=1$. To get some idea how the region $A_{\gamma}^{*}$ looks like for $k \geq 1$ we rewrite (3.14) and (3.15) in a different form. Define $w_{k}^{*}$ and $u_{k}^{*}$ as in P8 and P9 and put $v_{k}^{*}:=\sum_{j=0}^{k}\left|\beta_{k, j}^{*}\right|$. It can be checked by considering special cases and using P6-P8 that

where

$$
A_{\gamma}^{*}=B_{\gamma}^{*} \quad C_{\gamma}^{*}
$$

and

$$
B_{\gamma}^{*}=\left\{(x, y): \quad x \leq 0, \quad u_{k}^{*} x+\gamma v_{k}^{*}|y| \leq 0, \quad w_{k}^{*} x+\gamma v_{k}^{*}|y| \leq 2\right\}
$$

$$
C_{\gamma}^{*}=\left\{(\mathrm{x}, \mathrm{y}): \quad \mathrm{x} \leq 0, \quad \mathrm{v}_{\mathrm{k}}^{*}(-\mathrm{x}+\gamma|\mathrm{y}|) \leq \gamma-1\right\} \text {. }
$$

In view of $P 9$ the set $B_{\gamma}^{*}$ is empty for $k \geq 6$, therefore, Theorem 3 is applicable only for $k \leq 5$. It follows after some computations that the boundary of the region $A^{*}$ for $k=1,2,3,4$, and 5 is given by

$$
\mathrm{y}=\left\{\begin{array}{lr} 
\pm\left(2-\mathrm{w}_{\mathrm{k}}^{*}\right) /\left(\left(\mathrm{v}_{\mathrm{k}}^{*}\left(3-\left(\mathrm{w}_{\mathrm{k}}^{*}+\mathrm{v}_{\mathrm{k}}^{*}\right) \mathrm{x}\right)\right),\right. & 2 / \mathrm{w}_{\mathrm{k}}^{*} \leq \mathrm{x} \leq-1 / \beta_{\mathrm{k}, 1}^{*}, \\
\pm \mathrm{u}_{\mathrm{k}}^{*} \mathrm{x} /\left(\mathrm{v}_{\mathrm{k}}^{*}\left(\left(\mathrm{u}_{\mathrm{k}}^{*}+\mathrm{v}_{\mathrm{k}}^{*}\right) \mathrm{x}-1\right)\right), & -1 / \beta_{\mathrm{k}, 1}^{*} \leq \mathrm{x} \leq 0 .
\end{array}\right.
$$

Using the table of coefficients of Adams-Moulton methods given in Lapidus and Seifeld [23] we can compute $u_{k}^{*}, v_{k}^{*}$, and $w_{k}^{*}$ and these boundaries, for $k=1,2,3,4,5$, are plotted in Fig. 2. The coordinates of the points $Q_{k}^{*}\left(x_{k}^{*}, y_{k}^{*}\right), k=1,2,3,4,5$, are given in Table 2 below

\begin{tabular}{ccc}
\hline$k$ & $\mathrm{x}_{\mathrm{k}}^{*}$ & $\mathrm{y}_{\mathrm{k}}^{*}$ \\
\hline 1 & -2 & $2 / 5$ \\
2 & $-3 / 2$ & $36 / 119$ \\
3 & $-24 / 19$ & $88 / 425$ \\
4 & $-360 / 323$ & $4562 / 39232$ \\
5 & $-1440 / 1427$ & $303840 / 8845621$ \\
\hline
\end{tabular}

Table 2

As mentioned above, we were not able to apply the approach used in this paper to the general linear multistep methods with Hermite interpolation or high order Adams methods. 


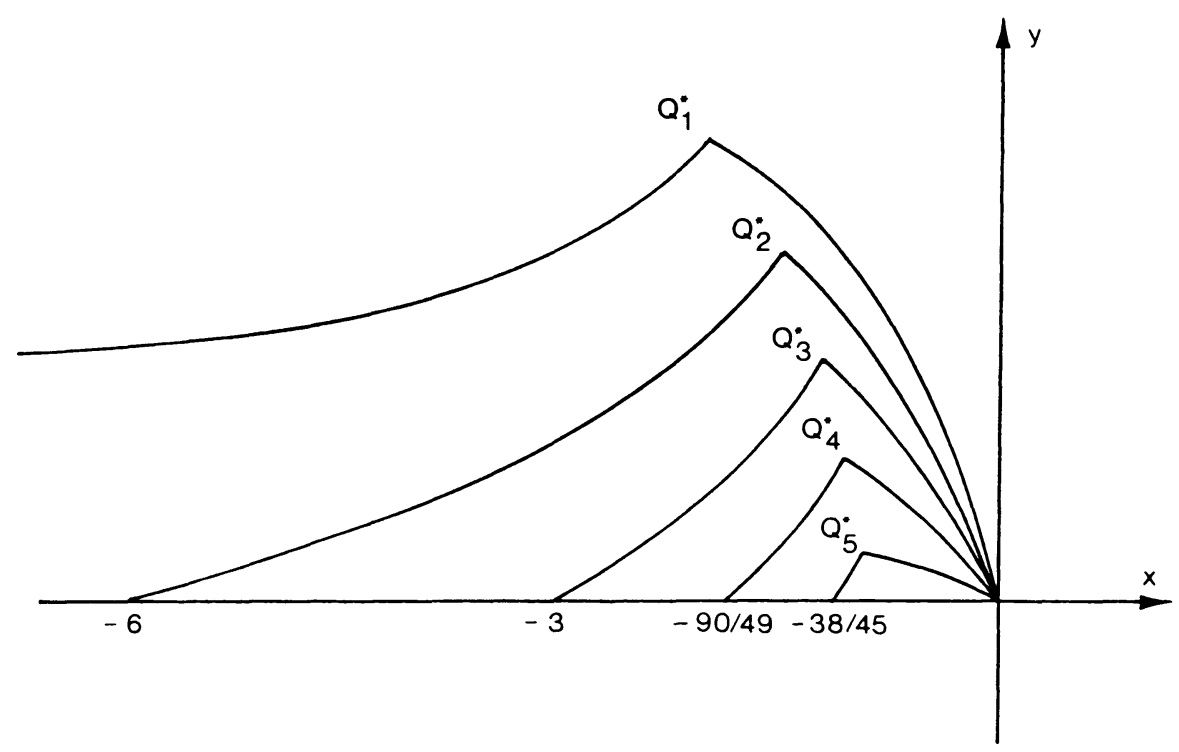

Fig. 2. Stability regions for Adams-Moulton methods for $k=1,2,3,4,5$.

REMARK. It was pointed out to us by Professor M. Zennaro that the main theorem in the paper by Jackiewicz [13] gives only sufficient, not necessary and sufficient condition for absolute stability of $\theta$-methods for delay differential equations. ACKNOWLEDGEMENT. This research was performed under Arkansas EPSCOR grant PRM-8011447.

\section{REFERENCES}

1. HALE, J. Theory of Functional Differential Equations, Springer Verlag, New York, 1977.

2. TAVERNINI, L. One step methods for the numerical solution of Volterra functional equations, SIAM J. Numer. Anal. 8 (1971), 786-795.

3. TAVERNINI, L. Linear multistep methods for the numerical solution of Volterra functional differential equations, Applicable Anal. 1 (1971), 169-185.

4. TAVERNINI, L. Numerical methods for Volterra functional differential equations, Invited paper, SIAM Fall Meeting, Madison, Wisconsin, 1971.

5. JACKIEWICZ, Z. Convergence of multistep methods for Volterra functional differential equations, Numer. Math. 32 (1979), 307-332.

6. JACKIEWICZ, Z. The numerical solution of Volterra functional differential equations of neutral type, SIAM J. Numer. Ana1. 18 (1981), 615-626.

7. JACKIEWICZ, $Z$. One step methods of any order for neutral functional differential equations, SIAM J. Numer. Anal. 21 (1984), 486-511.

8. ZVERKINA, T.S. A modified Adams' formula for the integration of equations with deviating argument, Trudy Sem. Teor. Differencial, Uravnenii s Otklon. Argumentom Univ. Druzby Narodov Patrisa Lumumby 3 (1965), 221-232 (In Russian).

9. BELLMAN, R. and COOKE, K.L. Differential-Difference Equations, Academic Press, New York, 1963.

10. CRYER, C.W. Highly stable multistep methods for retarded differential equations, SIAM J. Numer. Ana1. 11 (1974), 788-797.

11. BARWELL, V.K. Special stability problems for functional differential equations, BIT 15 (1975), 130-135.

12. VAN DER HOUWEN, P.J. and SOMMEIJER, B.P. Stability in linear multistep methods for pure delay equations, J. Comput. App1. Math. 10 (1984), 55-63. 
13. JACKIEWICZ, Z. Asymptotic stability analysis of $\theta$-methods for functional differential equations, Numer. Math. 43 (1984), 389-396.

14. WIEDERHOLT, L.F. Stability of multistep methods for delay differential equations, Math. Comp. 30 (1976), 283-290.

15. AL-MUTIB, A.N. Stability properties of numerical methods for solving delay differential equations, J. Comput. App1. Math. 10 (1984), 71-79.

16. OPPELSTRUP, J. RKFHB4 method for delay differential equations, In Numerical Treatment of Differential Equations (Ed. R. Bulirsch, R.D. Grigorieff and J. Schröder), Lectures Notes in Math. Vo1. 631, Springer Verlag, New York, 1978.

17. BICKART, T.A. F-stable and $F[\alpha, \beta]-$ stable integration interpolation methods in the solution of retarded differential-difference equations, J. Comput. Appl. Math. $\underline{8}(1982), 279-284$.

18. BRAYTON, R.K. and WILLOUGHBY, R.A. On the numerical integration of a symmetric system of a difference-differential equations, J. Math. Anal. App1. 18 (1967), 182-189.

19. FOX, L., MAYERS, D.F., OCKENDON, J.R. and TAYLER, A.B. On a functional differential equation, J. Inst. Math. App1. 8 (1971), 271-307.

20. KATO, T. and MCLEOD, J.B. The functional differential equation $y^{\prime}(x)=a y(\lambda x)+b y(x)$, Bull. Amer. Math. Soc. 77 (1971), 891-937.

21. HENRICI, P. Discrete Variable Methods in Ordinary Differential Equations, John Wiley, New York, 1962 .

22. HALL, G. Stability analysis of predictor-corrector algorithms of Adams type, SIAM J. Numer. Ana1. 11 (1974), 494-505.

23. LAPIDUS, L. and SEIfELD, J.H. Numerical Solution of Ordinary Differential Equations, Academic Press, New York, 1971. 


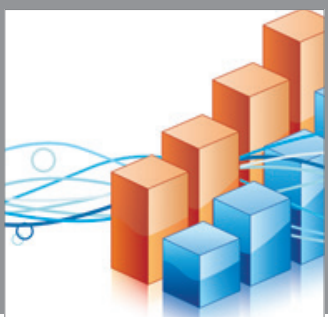

Advances in

Operations Research

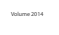

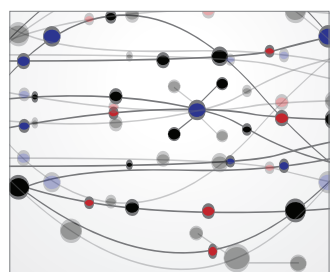

\section{The Scientific} World Journal
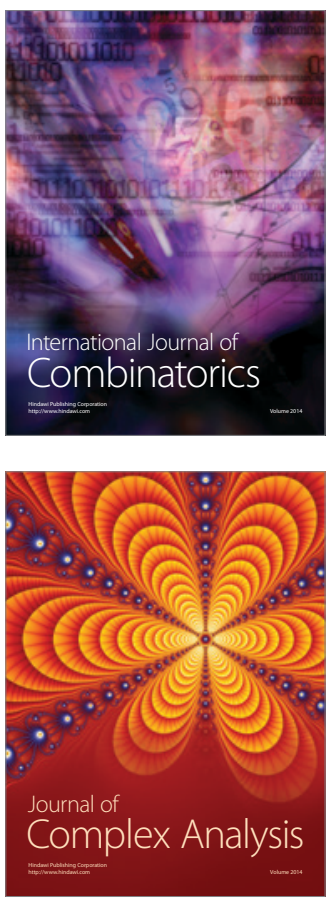

International Journal of

Mathematics and

Mathematical

Sciences
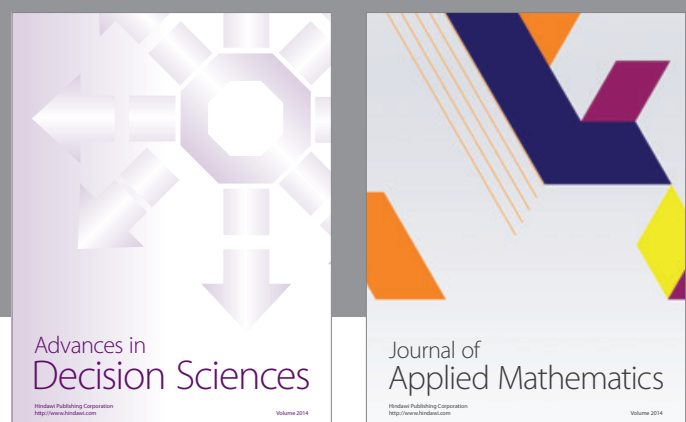

Journal of

Applied Mathematics
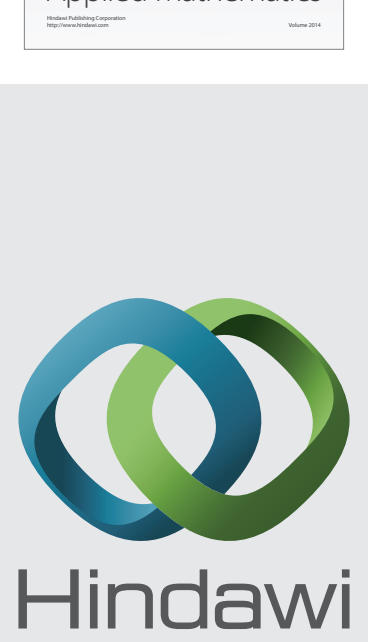

Submit your manuscripts at http://www.hindawi.com
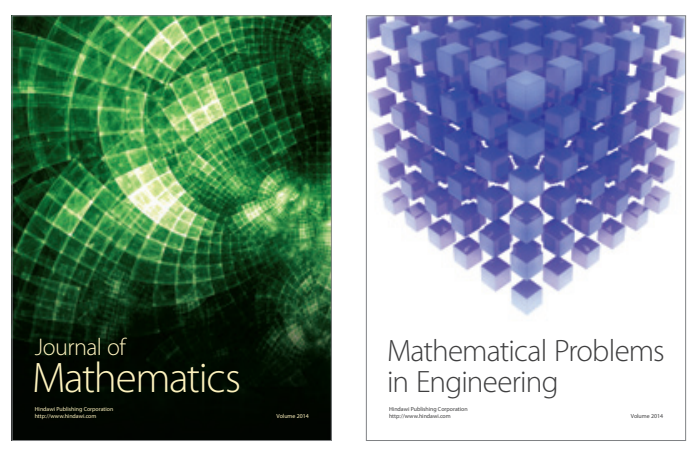

Mathematical Problems in Engineering
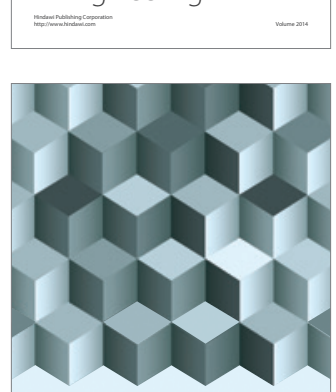

Journal of

Function Spaces
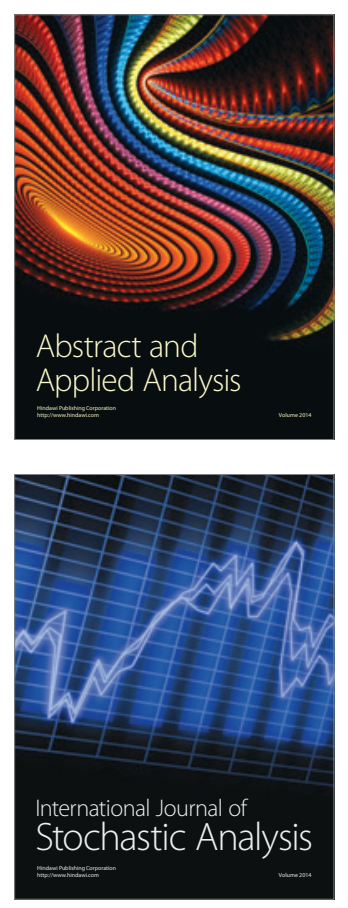

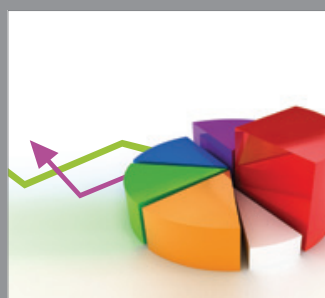

ournal of

Probability and Statistics

Promensencen
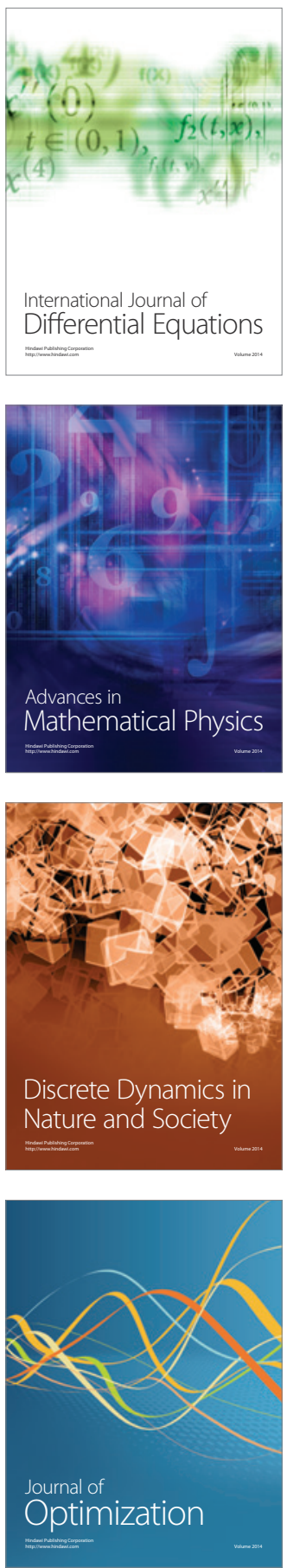